\title{
Produtividade Agregada Brasileira (1970-2000): Declínio Robusto e Fraca Recuperação
}

\author{
- Pedro Cavalcanti Ferreira* - Roberto Ellery JR. ** - Victor Gomes**
}

\begin{abstract}
RESUMO
Este artigo discute o comportamento da Produtividade Total dos Fatores (PTF) no Brasil entre 1970 e 1998. É feita uma análise de quanto da queda da PTF pode ser explicado a partir de mudanças na forma tradicional de cálculo desta variável. Entre as variações serão consideradas: utilização da capacidade instalada, modificações no uso do capital, mensuração do capital por meio do consumo de eletricidade, distorções no preço relativo, capital humano e investimento específico à determinada tecnologia. $O$ único caso no qual a PTF apresenta uma modificação em seu comportamento é o de correção de distorções no preço relativo, em que a PTF se recupera mais rapidamente.
\end{abstract}

\section{PalavRAS-CHAVE}

produtividade total dos fatores, progresso técnico, crescimento econômico

\section{ABSTRACT}

This study explores the productivity performance of the Brazilian economy between 1970 and 1998. We assess how much of the TFP downfall can be explained by some departures from the standard procedure. We incorporate to the standard measure utilization of capacity, changes in the workweek of capital, services of capital from electricity consumption, relative prices distortions, human capital, and investment in specific technology. We conclude that the downfall in productivity is quite robust to those specifications. The only case that presents a marked difference from the standard TFP measure occurs when relative prices of capital are corrected. The implications of this finding are a topic for future research.

KEYWORDS

total factor productivity, technical progress, economic growth

\author{
JEL CLASSIFICATION \\ 047
}

\footnotetext{
* Da EPGE da Fundação Getulio Vargas. Endereço para contato: EPGE - Fundação Getulio Vargas - Praia de Botafogo, 190 - Rio de Janeiro - RJ - 22253-900. E-mail: ferreira@fgv.bi.

* Do Departamento de Economia da Universidade de Brasília. Endereço para contato: Departamento de Economia, Universidade de Brasília - Campus Darcy Ribeiro, Asa Norte, Brasília DF - CEP: 70910-900. E-mails: ellery@unb. br; victorgomes@unb.br.

(Recebido em julho de 2005. Aceito para publicação em maio de 2007).
} 


\section{INTRODUÇÃO}

Após apresentar taxa média de crescimento do produto per capita de aproximadamente 4,4\% ao ano no período entre 1947 e 1979, a economia brasileira entrou em um longo processo de estagnação. A taxa média de crescimento do produto per capita entre 1980 e 2003 foi de apenas $0,63 \%$ ao ano.

Explicações usuais para essa mudança de comportamento costumam associá-la à crise da dívida externa nos anos 80 e ao descontrole monetário e fiscal que se seguiu a essa crise durante os anos seguintes. ${ }^{1}$ Apesar da teoria neoclássica tradicional relacionar o comportamento de longo prazo do produto per capita à trajetória da produtividade total dos fatores - PTF - (Solow, 1957), poucos trabalhos procuram relacionar a estagnação dos anos 80 e 90 com o comportamento da PTF.

Recentemente alguns artigos buscaram cobrir esta lacuna. Gomes, Pessôa e Veloso (2003) analisam o desempenho da economia brasileira em relação a outros países a partir da comparação da evolução da PTF. Bugarin et alli (2002) usam o modelo neoclássico para mostrar que o comportamento da economia brasileira nos anos $80 \mathrm{e}$ 90 pode ser explicado por choques de produtividade. Em todos esses artigos a PTF é calculada a partir de estimativas para o estoque de capital e de horas trabalhadas e de uma função de produção agregada.

As estimativas de estoque de capital são construídas geralmente através da acumulação do investimento, mas esse método apresenta uma série de problemas como descrito em Pritchett (2000). Da mesma forma, problemas relacionados ao uso do capital costumam ser desconsiderados no cálculo da PTF em vários trabalhos aplicados.Uma vez que a PTF é calculada como um resíduo, estes problemas relacionados à mensuração dos insumos podem implicar um grande viés na série estimada de produtividade.

Tendo o problema de mensuração dos insumos em mente, neste trabalho são feitos vários cálculos da PTF de forma a considerar diversas medidas alternativas da produtividade. Como no trabalho de Ohanian (2001), será avaliado quanto da queda da PTF pode ser explicado por diferentes medidas das variáveis relevantes, principalmente em relação ao capital, já que este é o fator que potencialmente apresenta mais problemas de mensuração. Neste sentido, este artigo estuda em essência a robustez da queda da PTF em relação a fatores tais como a utilização da capacidade, semana de trabalho do capital, serviços do capital medido a partir do consumo de energia elétrica, capital humano, capital usado na produção, entre outros. Além dessas medidas de resíduos de Solow, também calculamos a produtividade a partir da hipótese de progresso técnico específico aos equipamentos (ver Greenwood, Hercowitz e Krussell, 1997).

1 Como exemplo desta interpretação, ver a compilação de artigos de Abreu (1990). 
Uma característica deste trabalho é que, assim como em Gomes, Pessôa e Veloso (2003), a PTF será analisada em termos relativos. Este é um diferencial em relação a outros estudos como os de Bonelli e Fonseca (1998) e Pinheiro et alli (2001), que não comparam a PTF do Brasil com a da economia líder, os Estados Unidos. Como a riqueza, a PTF é um conceito relativo, portanto é importante estudar seu comportamento no Brasil comparativamente a outras economias mais avançadas. Neste estudo, em particular, utilizaremos a PTF americana como norma, pois esta economia foi a líder de crescimento econômico no século 20.

O principal resultado do artigo é que, ao comparar as diversas medidas, percebe-se que o comportamento da produtividade no Brasil é bastante robusto. Isto significa que considerar fatores como uso do capital, medidas alternativas para o capital e variações no capital humano não leva a mudanças significativas no comportamento da PTF. De fato, apenas com a correção de distorção dos preços relativos é possível notar mudanças relevantes. Uma conclusão imediata deste resultado é que mudanças na forma de cálculo da PTF não devem gerar mudanças significativas no fato de que seu comportamento explica de forma razoável o comportamento do produto per capita no Brasil.

Na próxima seção será analisado o caso base de medida da PTF para o Brasil. $\mathrm{Na}$ seção 3 serão realizadas estimativas da produtividade considerando a utilização do capital, a semana de uso do capital e, também, medindo o capital a partir do consumo de energia elétrica. Na seção 4 será apresentada a estimativa de PTF com capital humano e, na seção 5, apresentamos medidas apenas com máquinas e equipamentos e também sem estruturas residenciais. Na seção 6 serão tratados os problemas de deflacionamento relativos à mensuração do estoque de capital no Brasil. Na seção 7 será apresentada uma medida da PTF a partir da idéia de progresso tecnológico específico ao investimento. Finalmente, a seção 8 apresenta as considerações finais do trabalho.

\section{MEDINDO A PRODUTIVIDADE: O CASO BASE}

O caso base para analisar a PTF utiliza dados de capital e trabalho comuns na literatura sobre ciclos econômicos e crescimento, bem como a função de produção CobbDouglas, dada por:

$$
Y_{t}=K_{t}^{\theta}\left(A_{t} L_{t}\right)^{1-\theta}
$$


onde $Y_{t}$ representa o produto agregado, $K_{t}$ representa o estoque de capital, $L_{t}$ é o insumo de trabalho e $A_{t}$ é a produtividade total dos fatores.

Para a escolha do valor do parâmetro $\theta$, que representa a participação da renda do capital na renda nacional, foram utilizadas as evidências encontradas por Gomes, Bugarin e Ellery Jr (2005) e Gomes, Lisboa e Pessôa (2002). Estes estudos seguem a proposta de Gollin (2002) para corrigir possíveis problemas de mensuração da participação do capital. Desta forma o valor usado para o parâmetro $\theta$ será de 0,40 .

A medida de produto escolhida foi o PNB; por sua vez, o estoque de capital foi construído a partir da série de investimento do IBGE $\left(X_{t}\right)$, assumindo uma taxa de depreciação de aproximadamente $9 \%^{2}$, e usando a regra de movimento do estoque de capital dada por:

$$
K_{t+1}=(1-\delta) K_{t}+X_{t}
$$

tal que $\delta$ é a taxa de depreciação do capital. Para evitar problemas relacionados à estimativa do estoque inicial de capital, a série foi construída a partir de 1950, mas só foram utilizados dados posteriores a 1970 para nossas estimativas. A série de trabalho foi construída como o total de horas trabalhadas, obtido por meio dos dados agregados da PNAD. No apêndice apresentamos maiores detalhes dos dados e da metodologia.

A Figura 1 apresenta o nosso caso base. A linha tracejada é a PTF e a linha sólida representa a PTF descontada a tendência de crescimento da economia americana no século 20. A taxa de crescimento da PTF para os EUA, $g_{U S}$, foi de $1.44 \%$ ao ano e para retirar a tendência foi usado o seguinte fator: $\left(1+g_{U S}\right)^{t-1969}$, onde $t=1970,1971, \ldots$, 1998. O pico da produtividade foi em 1973, e desde então ela cai até atingir menos do que 60\% do nível de 1980 e 54\% do nível de 1970 (ver Tabela 1). Esta queda da PTF a partir dos anos 70 está amplamente documentada em outros trabalhos que utilizam metodologia semelhante à nossa e pode ser hoje considerada um fato estilizado da economia brasileira. ${ }^{3}$

2 Este valor da depreciação é utilizado por diversos autores em contexto semelhante. Ver, por exemplo, Kydland e Prescott (1982), Kidland e Zarazaga (2002) e Prescott (2002).

3 Ver, por exemplo, Gomes, Pessôa e Veloso (2003) e Bugarin et alli (2002). 
FIGURA I - PTF - CASO BASE

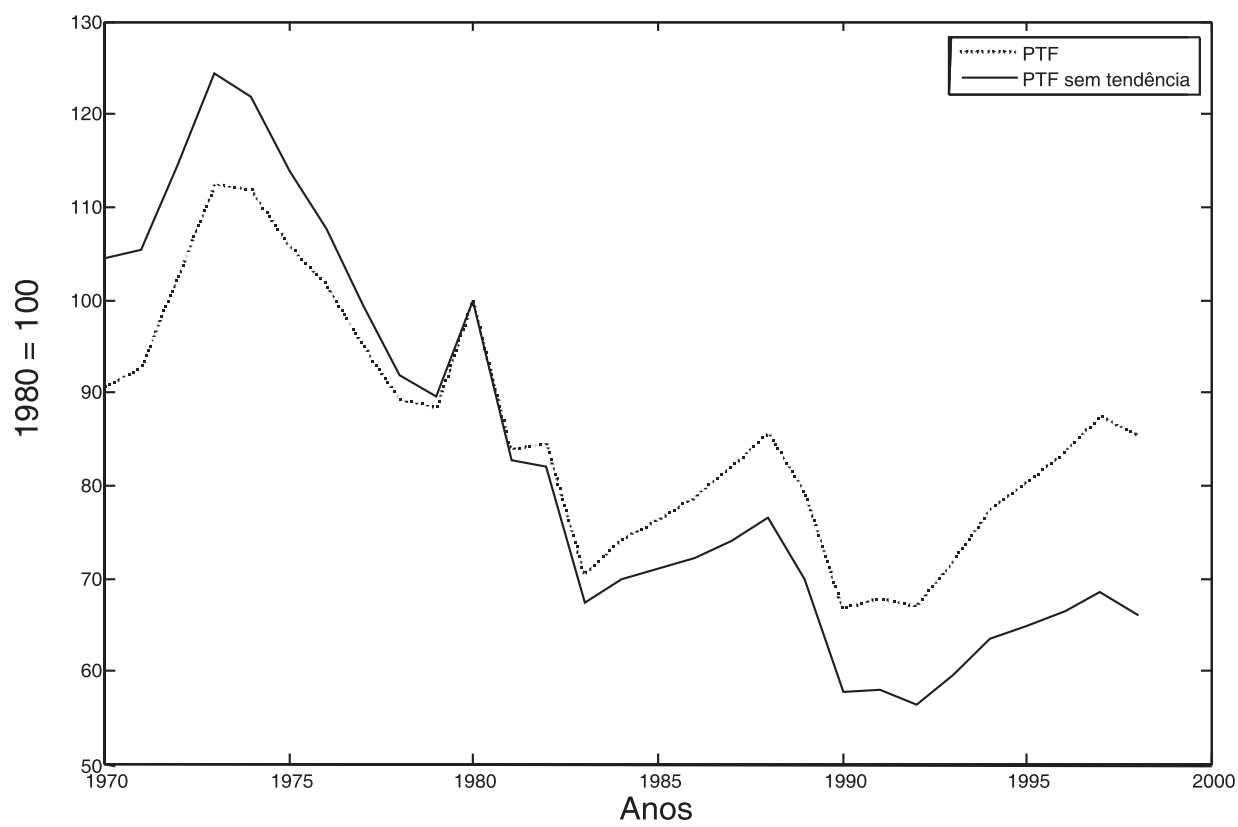

Nas próximas seções serão apresentadas medidas alternativas para a PTF de forma a estudar a robustez deste resultado. Em cada caso será feita uma comparação com os dados apresentados na Figura 1.

\section{UTILIZAÇÃO DOS FATORES}

A formulação geral da função de produção que contempla o serviço dos insumos é a seguinte:

$$
Y_{t}=A_{t}^{1-\theta}\left(\mu_{t} K_{t}\right)^{\theta}\left(\varphi_{t} L_{t}\right)^{1-\theta}
$$

tal que $\mu_{t} K_{t}$ e $\varphi_{t} L_{t}$ são os serviços do capital e do trabalho, respectivamente, enquanto $0<\mu_{t}<1$ e $0<\varphi_{t}<1$ são as taxas de utilização de cada fator. Usando esta especificação, serão analisadas três possibilidades: (i) taxa de utilização do capital, (ii) a semana de trabalho do capital e do trabalho, e (iii) os serviços do capital medido pelo consumo de energia elétrica. 
No primeiro caso $\mu_{t}$ representa a taxa de utilização do capital. A série de utilização do capital é o índice anual de utilização do capital da Fundação Getúlio Vargas. Para o insumo trabalho é feita a hipótese de que o total de horas trabalhadas é igual a $\varphi_{t} L_{t}$.

Na Figura 2 e na Tabela 1 são mostrados os resultados da mensuração da PTF com utilização da capacidade. O principal resultado da aplicação do uso da utilização do capital é que a PTF é menos volátil do que no caso base e permanece, em média, três pontos acima da PTF da seção anterior. Por exemplo, o valor mínimo no caso base é 54,06 , passando a 60,66 abaixo da tendência no caso com utilização do capital.

\section{FIGURA 2 - PTF SEM TENDÊNCIA E COM UTILIZAÇÃO DOS FATORES}

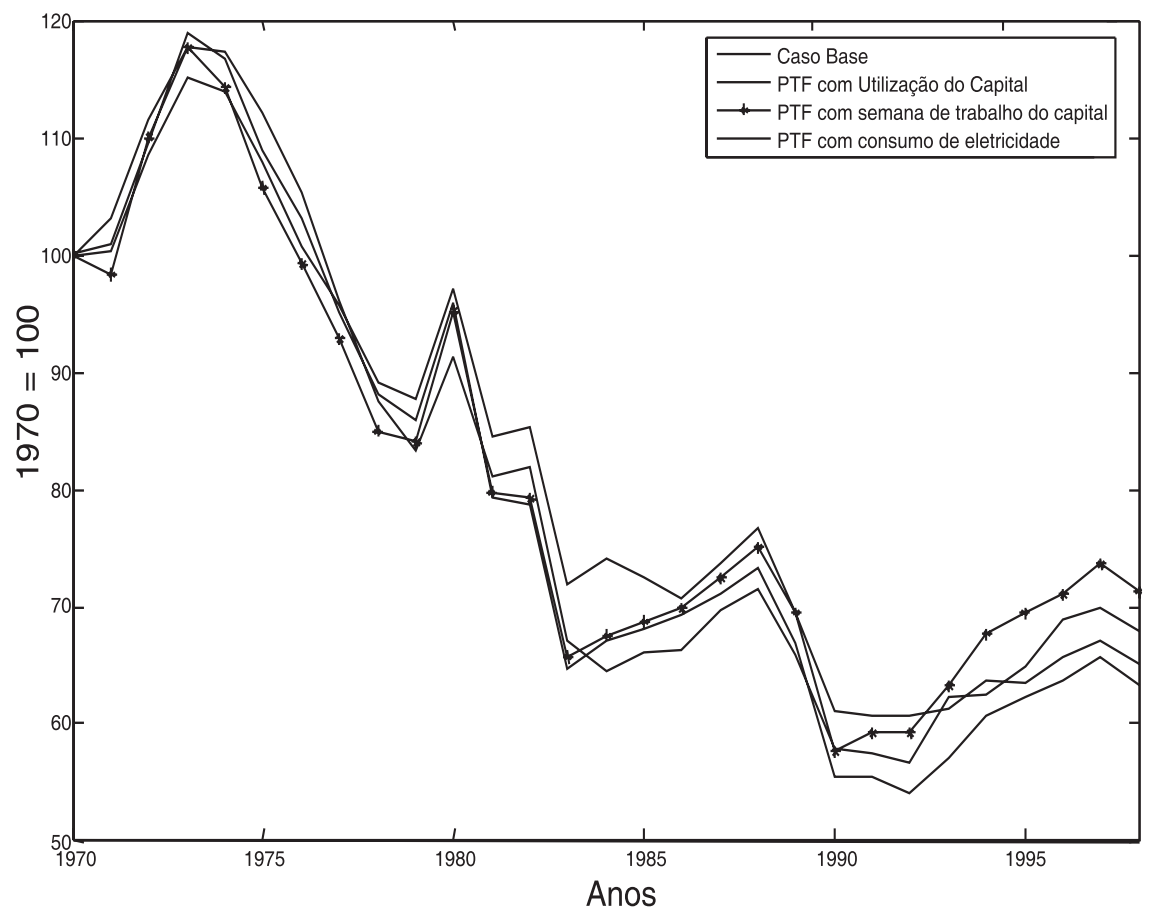

O próximo caso alternativo é a semana de trabalho do capital. Será usada a estratégia de supor que o uso do capital é proporcional à semana de trabalho dos trabalhadores. Os equipamentos e as construções são utilizados apenas quando existem trabalhadores para operá-los. Este caso é o analisado, entre outros, por Prescott (1998). Neste caso, uma semana de trabalho de tamanho diferente é um fator de produção diferente. De forma simples, $N_{h}$ é a medida da semana de trabalho de tamanho $h \in H \subset(0,1]$ e $N$ é um vetor de dimensão finita. Para cada tamanho da semana de trabalho a função de produção agregada é $F_{h}\left(K_{h}, M_{h}\right)=h A_{h}^{1-\theta} K_{h}^{\theta} M_{h}^{1-\theta}$. A função agregada de produção 
é obtida somando sobre $h$ e igualando os produtos marginais do capital entre estas tecnologias agregadas. Esta função pode ser representada da seguinte forma:

$$
Y_{t}=N_{t} A_{t}^{1-\theta} K_{t}^{\theta} M_{t}^{1-\theta}
$$

onde $M_{t}$ representa a semana de trabalho e $N_{t}$ o emprego. O total de horas trabalhadas é igual à semana de trabalho multiplicado pelo total de empregados.

Para medir a PTF a partir da equação (4), foram utilizadas as séries de semana de trabalho média e de emprego (para mais detalhes, ver Apêndice). Os resultados desta especificação da produtividade medida com a função de produção com a semana de trabalho são muito próximos aos obtidos no caso base. A única diferença sensível é que a PTF nos anos 90 é dois ou três pontos superior à observada no caso base.

TABELA I - PTF SEM TENDÊNCIA, CASO BASE, UTILIZAÇÃO DOS FATORES E CAPITAL HUMANO, $1970=100$

\begin{tabular}{lccccc}
\hline & & \multicolumn{4}{c}{ PTF medida com: } \\
\cline { 3 - 6 } Anos & Caso Base & $\begin{array}{c}\text { Utilização do } \\
\text { Capital }\end{array}$ & $\begin{array}{c}\text { Semana de } \\
\text { Trabalho }\end{array}$ & $\begin{array}{c}\text { Consumo de } \\
\text { Eletricidade }\end{array}$ & Capital Humano \\
\hline 1971 & 100,87 & 100,29 & 98,25 & 103,01 & 101,40 \\
1975 & 108,91 & 107,86 & 105,68 & 112,20 & 111,79 \\
1980 & 95,72 & 97,05 & 95,12 & 91,29 & 97,32 \\
1985 & 68,02 & 72,46 & 68,65 & 65,99 & 67,13 \\
1990 & 55,38 & 61,10 & 57,58 & 57,90 & 52,34 \\
1995 & 62,11 & 63,47 & 69,35 & 64,81 & 56,71 \\
1998 & 63,15 & 65,06 & 71,27 & 67,83 & 56,18 \\
\hline
\end{tabular}

Outra alternativa é medir os serviços do capital a partir do consumo de energia elétrica. A idéia é que quando se usa a máquina e a estrutura produtiva também se usa eletricidade e, desta forma, está se usando os serviços do capital; portanto, o consumo de energia elétrica industrial seria uma boa aproximação ao uso do fator capital na função de produção. Por outro lado, um problema com esta estratégia é a adoção de equipamentos que poupem energia elétrica, pois daria a falsa impressão de queda no uso do serviço do capital. Na Figura 3 é apresentada a razão entre o consumo de energia elétrica industrial e pelo consumo de máquinas e equipamentos. 


\section{FIGURA 3 - CONSUMO DE ELETRICIDADE POR MÁQUINA E EQUIPAMENTO, $1970=100$}

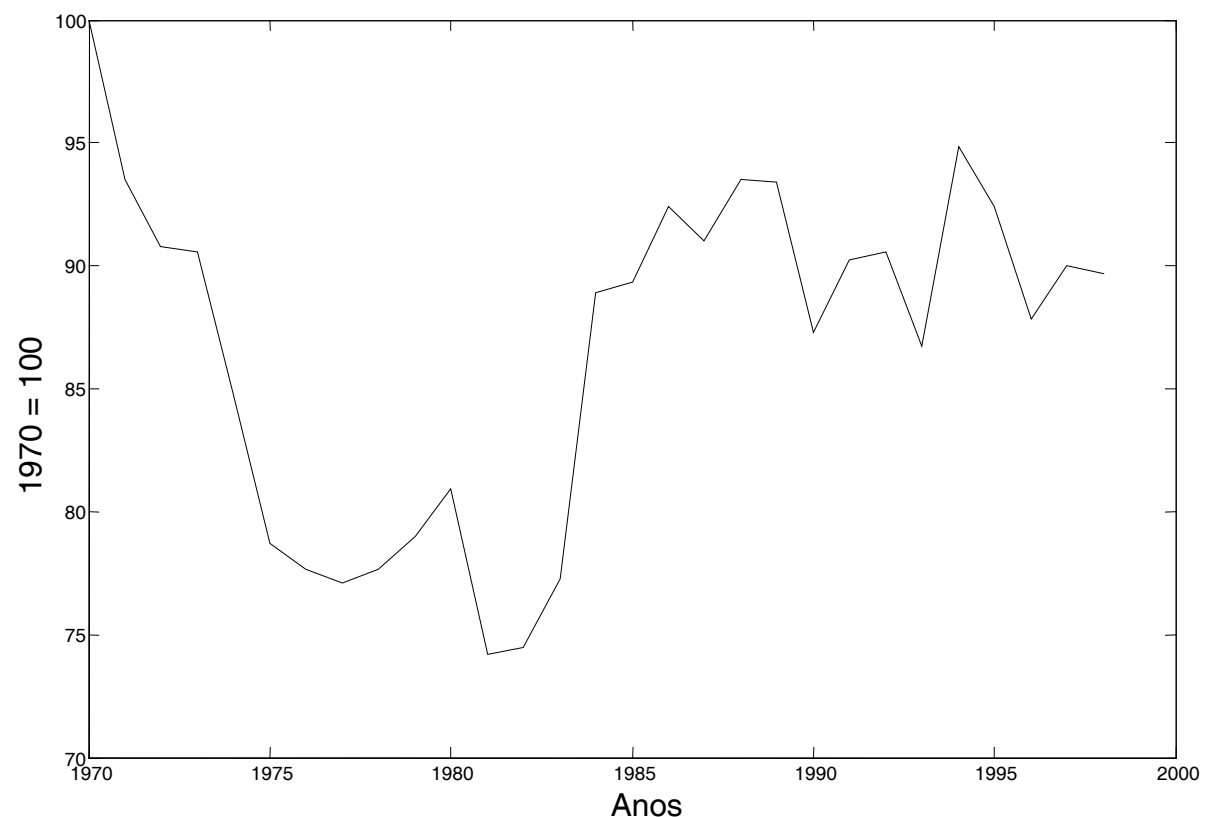

Na Figura 3 é possível observar que a razão eletricidade-capital caiu até 1977, mas entre 1983 e 1985 este número aumenta rapidamente, cerca de 15 pontos. ${ }^{4}$ Portanto, as medidas de PTF nos anos 70 e começo dos anos 80 podem ser mais baixas do que o esperado devido ao comportamento do consumo de energia elétrica industrial. Abstraindo dos problemas relacionados acima, suponha que o consumo de energia elétrica seja proporcional ao uso do capital e dado por $E_{t}=\mu_{t} K_{t} \cdot{ }^{5}$ Este caso mostra um padrão similar aos anteriores. Esta medida de PTF mostra o nível da produtividade abaixo do caso base no meio dos anos 80 , mas nos 90 a produtividade se recupera e fica de 4 a 5 pontos acima do caso base. A diferença, portanto, não é relevante.

4 O aumento do consumo de energia elétrica nos anos 80 coincide com o começo de operação das hidroelétricas de Tucuruí e Itaipu. A importância destes projetos é enorme. Por exemplo, em 2000 Itaipu contribuiu com $24 \%$ da oferta total de energia elétrica. De acordo com as informações de Ferreira e Malliagros (1999), a capacidade nominal do Brasil aumentou aproximadamente $50 \%$ entre 1980 e 1990.

5 Para medir o consumo de energia elétrica foi utilizada a série de consumo do Ministério das Minas e Energia para os anos de 1970 a 1998. Para mais detalhes, ver Apêndice. 


\section{CAPITAL HUMANO}

Outro fator que pode afetar a produtividade total dos fatores é a acumulação de capital humano, que até agora foi ignorada em nossos cálculos. Entretanto, talvez hoje seja mais comum calcular PTF com alguma medida de capital humano do que sem ela. Para mensurar a produtividade com capital humano foi usada a função de produção proposta por Bils e Klenow (2000). Para modelar capital humano os autores usam uma formulação minceriana de retorno da escolaridade. A hipótese-chave é que o nível de habilidade de um trabalhador com $\eta$ anos de escolaridade é $e^{\varphi \eta}$ maior do que o nível de habilidade de um trabalhador sem nenhuma educação.

Estas hipóteses levam à seguinte função de produção:

$$
Y_{t}=A_{t}^{1-\theta} K_{t}^{\theta}\left(H_{t} L_{t}\right)^{1-\theta}=A_{t}^{1-\theta} K_{t}^{\theta}\left(e^{\varphi \eta_{t}} L_{t}\right)^{1-\theta}
$$

onde $\eta_{t}$ será calculado como os anos médios de escolaridade e o parâmetro $\phi$ será definido como 0,08, valor estimado em Ferreira, Issler e Pessôa (2004). Para a série de escolaridade foram utilizados os dados de Barro e Lee (2000), interpolados entre qüinqüênios.

\section{FIGURA 4 - PTF SEM TENDÊNCIA E COM CAPITAL HUMANO}

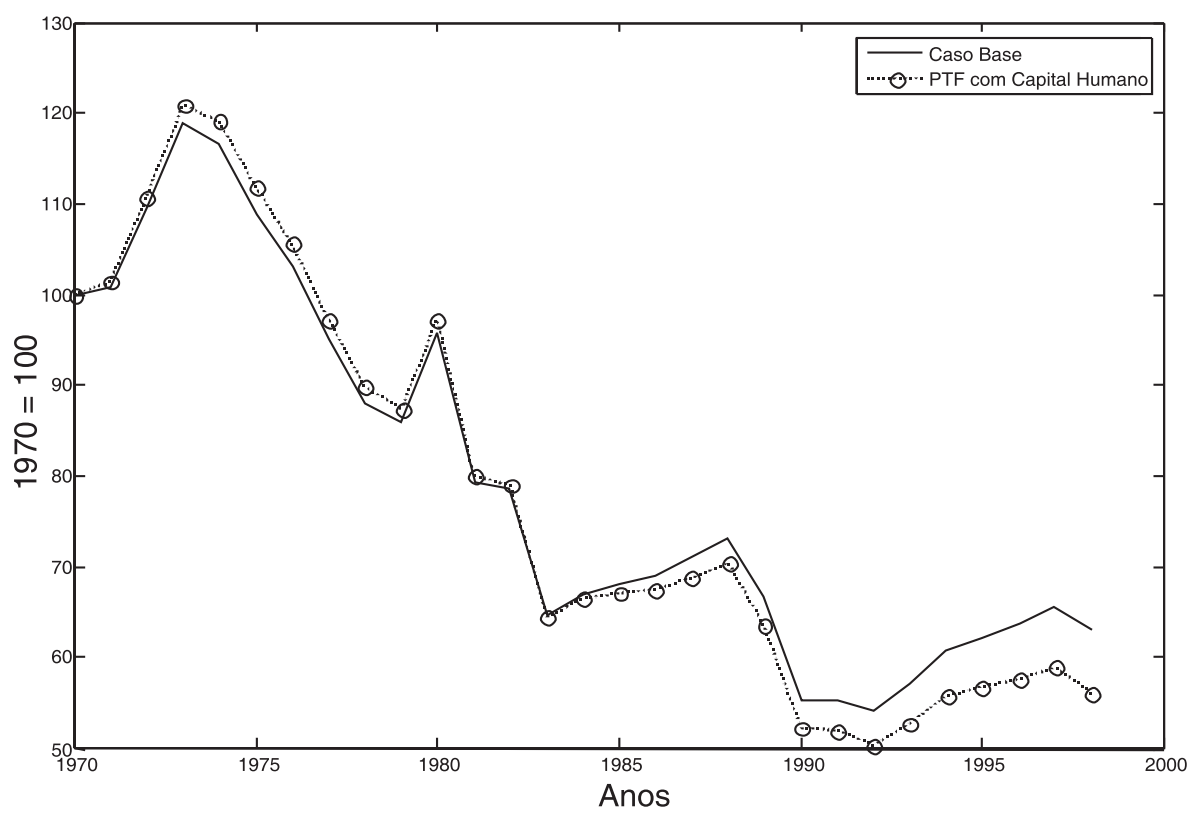


A Figura 4 mostra o gráfico da PTF medida a partir da equação (5) e o caso básico. $\mathrm{Na}$ Tabela $\mathrm{l}$ as duas séries são também apresentadas. Como os anos de escolaridade média cresceram de forma sustentada entre 1970 e 1998, a introdução do capital humano aumenta a queda da produtividade nos anos 90. A partir do meio dos anos 80 até os anos 90, a PTF medida com capital humano cai mais do que o caso base e alcança o seu menor valor em 1992, que foi de 50,39.

\section{MEDIDAS ALTERNATIVAS PARA O CAPITAL}

Uma questão comumente adotada em procedimentos de estimação de funções de produção é a de que só devem ser considerados os insumos efetivamente utilizados na produção de bens e serviços. É esta estratégia que será adotada nesta seção, tendo como propósito obter uma medida de produtividade mais associada à tecnologia utilizada pelas plantas produtivas. Para realizar esta tarefa serão propostas mais duas medidas de produtividade. Na primeira, o capital será composto apenas por máquinas e equipamentos; na segunda, será construído um estoque de capital composto por máquinas e equipamentos mais as construções não-residenciais. Para garantir a consistência desta última estimativa, os pagamentos de aluguéis foram deduzidos do PNB.

\section{FIGURA 5 - PTF SEM TENDÊNCIA, CÁLCULO COM MÁQUINAS E EQUIPAMENTOS E COM A ADIÇÃO DE CONSTRUÇÕES NÃO- RESIDENCIAIS}

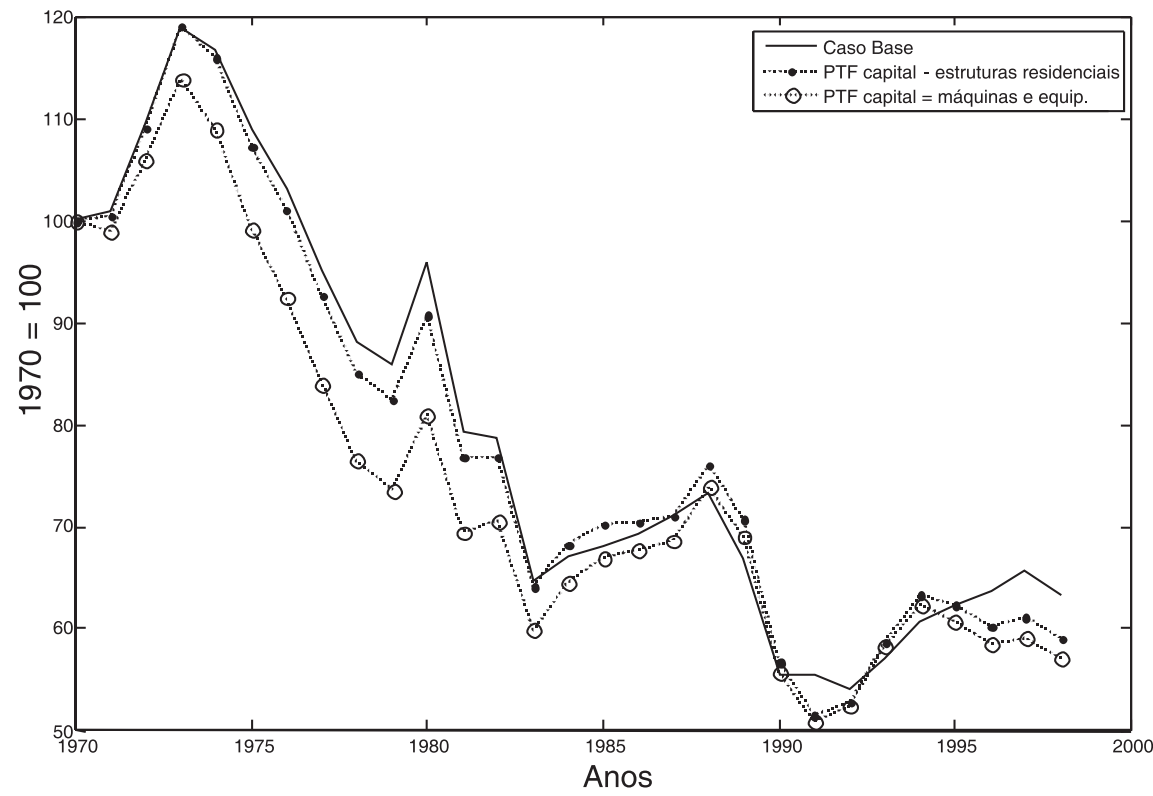


A Figura 5 e a Tabela 2 apresentam estas novas medidas de PTF juntamente com o caso base. Em termos gerais as medidas desta seção são menores que o caso base nos anos 70, algo em torno de 7 a 9 pontos. Nos anos 80 esta medida alcança o caso base e nos anos 90 permanece, aproximadamente, 3 pontos abaixo do caso base.

TABELA 2 - TFP SEM TENDÊNCIA, CASO BASE E 'CAPITAL PRODUTIVO'

\begin{tabular}{lcccc}
\hline Anos & Caso Base & $\begin{array}{c}\text { Ponstrução não-residencial }+ \\
\text { máquinas e equip. }\end{array}$ & $\begin{array}{c}\text { Máquinas e } \\
\text { Equipamentos }\end{array}$ & $\begin{array}{c}\text { Ajuste de Preços } \\
\text { Relativos }\end{array}$ \\
\hline 1971 & 100,87 & 100,41 & 98,93 & 109,64 \\
1975 & 108,91 & 107,23 & 99,11 & 132,97 \\
1980 & 95,72 & 90,65 & 81,02 & 113,10 \\
1985 & 68,02 & 70,23 & 66,99 & 83,11 \\
1990 & 55,38 & 56,71 & 55,62 & 73,75 \\
1995 & 62,11 & 62,22 & 60,74 & 92,01 \\
1998 & 63,15 & 59,00 & 57,04 & 95,35 \\
\hline
\end{tabular}

O segundo caso é aquele no qual o estoque de capital é definido como a soma das construções não-residenciais com máquinas e equipamentos. Mais uma vez os valores encontrados são semelhantes aos do caso base.

\section{AJUSTE DOS PREÇOS RELATIVOS}

As estimativas do estoque de capital no Brasil podem ser afetadas pelo grande aumento no preço relativo das construções no fim dos anos 80. Isto pode superestimar o valor do estoque de capital e, conseqüentemente, causar uma subestimação da produtividade total dos fatores. A Figura 6 mostra o comportamento do preço relativo da construção e das máquinas e equipamentos. ${ }^{6}$ Note-se que, enquanto o preço relativo das máquinas e equipamentos cai no período entre 1970 e 2000 , o preço relativo das construções aumenta, principalmente nos anos 80 .

6 O índice de preços utilizado foi o IPA e seus componentes para máquinas e equipamentos e material de construção. Para mais detalhes, ver Apêndice. 
FIGURA 6 - PREÇOS RELATIVOS DAS CONSTRUÇÕES E DAS MÁQUINAS E EQUIPAMENTOS
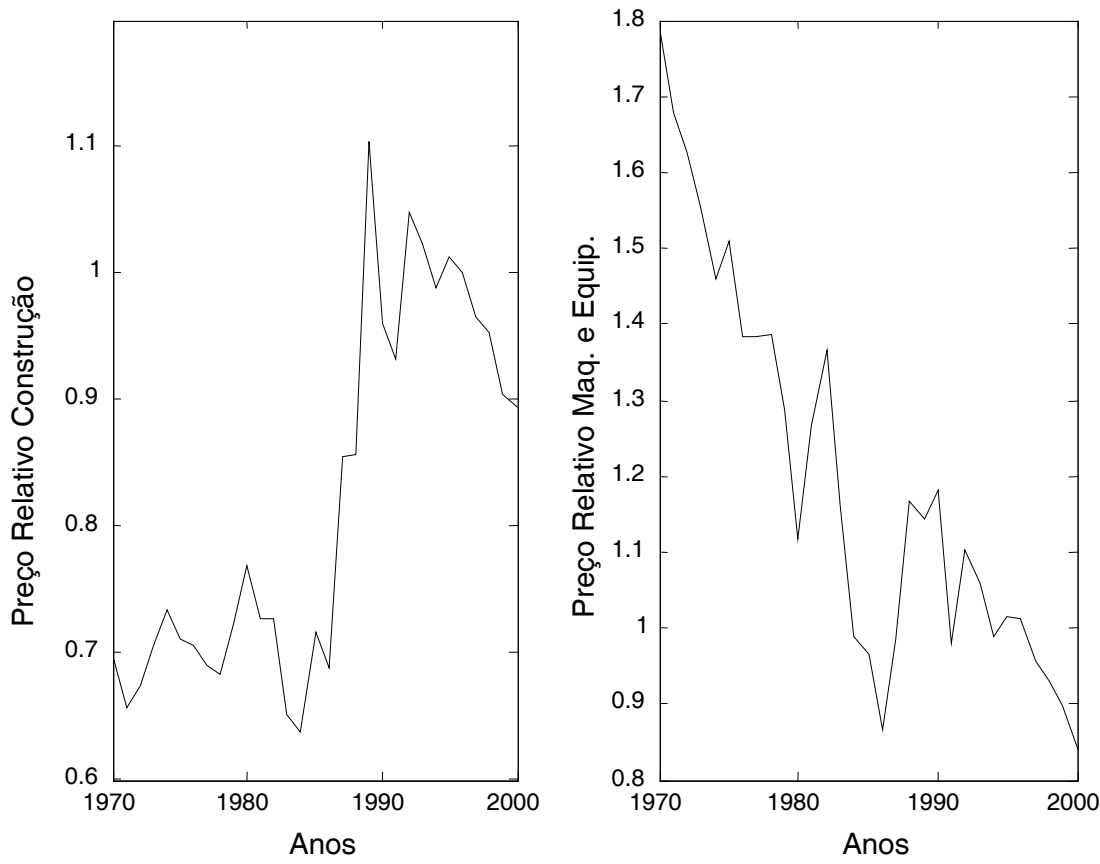

Para tratar deste problema, Bugarin et alli (2002) construíram uma série de estoque de capital em que os investimentos em máquinas e equipamentos e construções são deflacionados por um índice de preços específico para cada um. Uma série de capital construída da mesma forma será utilizada para calcular uma nova estimativa para PTF. A Figura 7 ilustra a série de capital agregado por pessoa em idade de trabalho corrigida por preços relativos e a série de capital sem esta correção. Como se pode observar, a série corrigida mostra que o estoque de capital pára de crescer durante os anos 80, cai ligeiramente no começo dos 90 e depois se recupera. Em contraste, a série não corrigida, utilizada no caso base (e em grande parte dos estudos do gênero no Brasil), cresce continuamente em todo o período. 
FIGURA 7 - ESTOQUE DE CAPITAL POR PESSOA EM IDADE DE TRABALHO (10-69 ANOS)

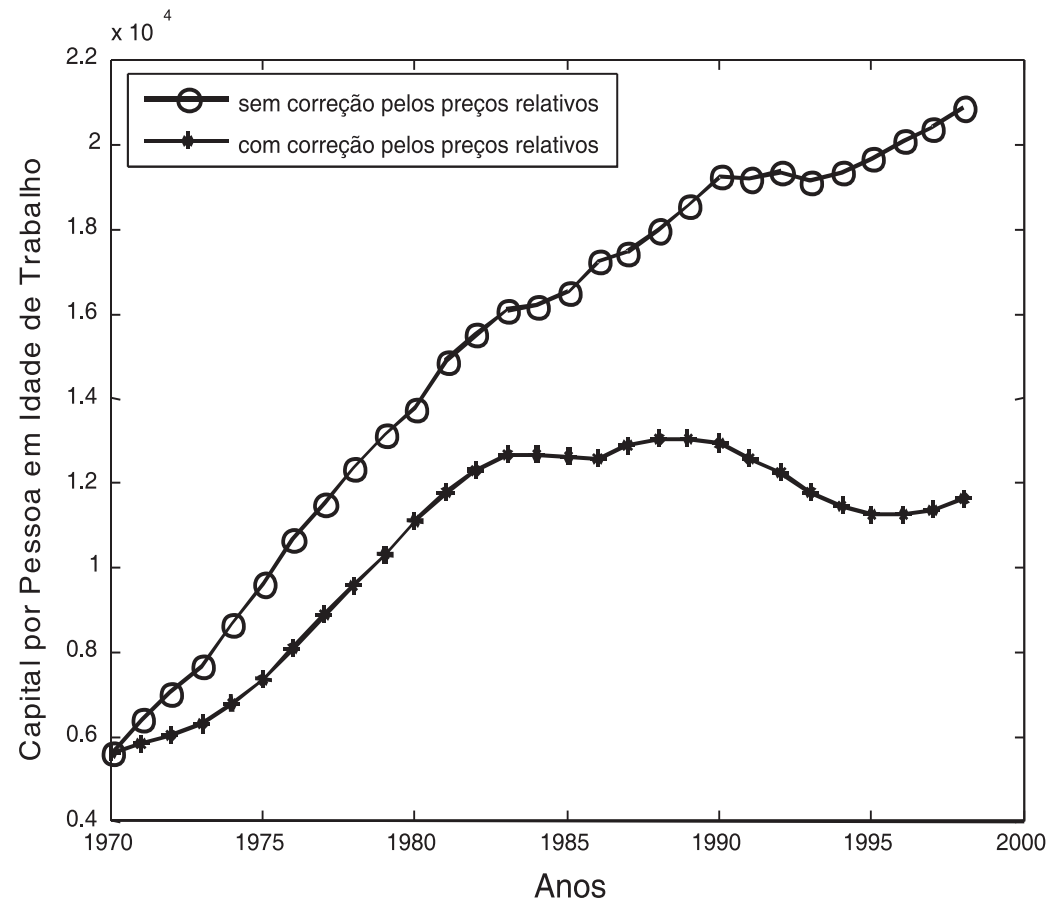

Na Figura 8 e na Tabela 2 esta nova série é apresentada. A nova medida da PTF apresenta um comportamento diferente das demais, como se poderia esperar, dado o comportamento diferenciado do capital. Tanto o seu valor máximo quanto o seu valor mínimo são 20 pontos superiores aos apresentados no caso base. De fato a nova série é consistentemente maior do que a série do caso base. 


\section{FIGURA 8 - PTF SEM TENDÊNCIA E COM O CAPITAL CORRIGIDO PELOS PREÇOS RELATIVOS}

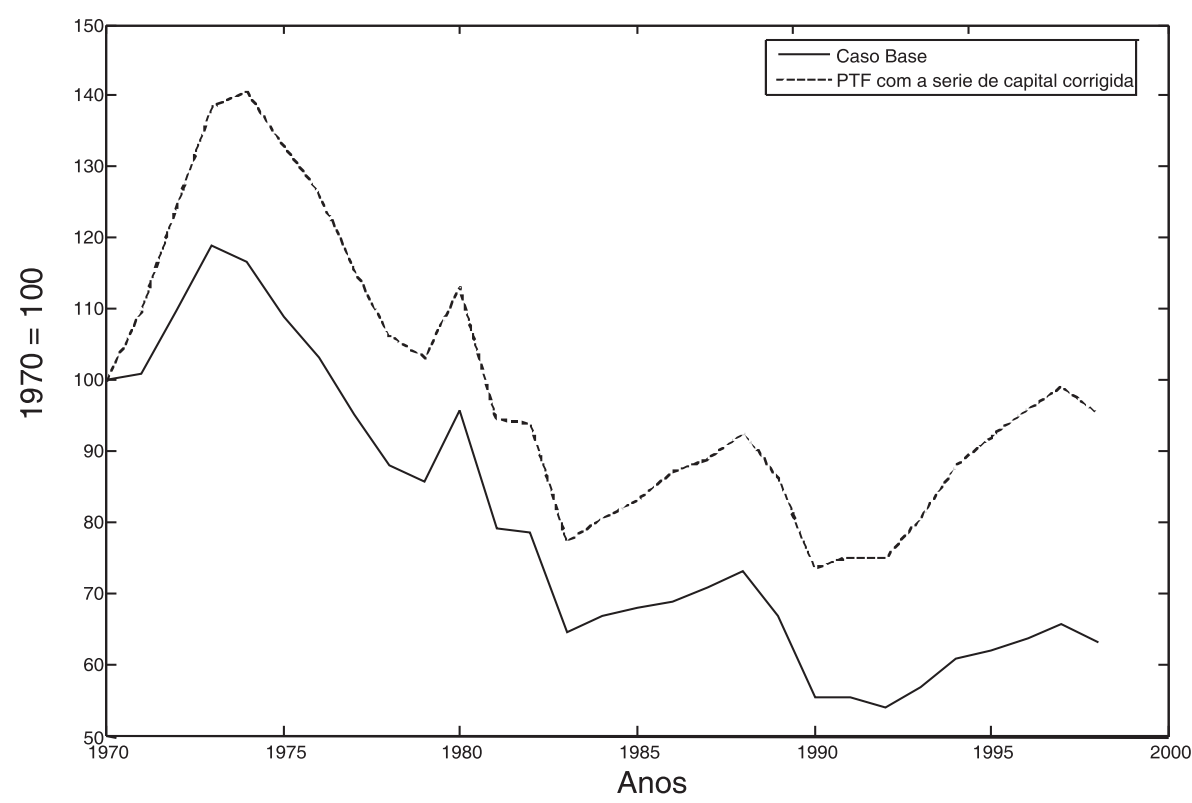

Dentre os principais pontos a serem ressaltados estão os fatos de que o ponto mais baixo em 1984 está em torno de $80 \%$ abaixo da tendência e aumenta para 92,62 em 1998. Como no caso base, há uma acentuada queda entre 1975 e 1984. Após a segunda queda da produtividade em 1992, o crescimento da PTF é rápido, fazendo com que a mesma volte para a tendência em 1997. Note-se que este é o único caso no qual a PTF descontada retorna aos níveis de 1970, ou seja, a taxa de crescimento da produtividade total dos fatores no Brasil entre 1970 e 1998 não seria inferior à da economia americana.

O fato de que a medida da PTF pode ser sensível a correçôes de preços relativos foi apontado por Jorgenson e Griliches (1967). Nesse trabalho, os autores mostram que a correção por preços relativos altera a forma como a PTF explica o comportamento do produto. O motivo para esta mudança no papel da PTF é que, ao não considerar alterações nos preços relativos, mudanças que ocorrem no uso dos serviços dos fatores de produção são creditadas à PTF, visto que esta é calculada como resíduo. No caso em discussão, o aumento do preço relativo do capital faz com que a análise do valor do estoque de capital superestime a quantidade de capital. Como resultado, com o estoque de capital inflado, a PTF parece ser menor do que realmente é, já que o preço relativo das construções subiu por um longo período e este efeito foi repassado para a taxa de crescimento da PTF. 
No cálculo da PTF utiliza-se o valor do estoque de capital como medida do serviço de capital. Definindo $p$ como o nível geral de preços e $p_{K}$ como o preço capital e K o valor nominal do estoque de capital, a medida do capital real será dada por $K^{*}=\frac{\mathrm{K}}{p_{K}}$; entretanto, a medida usada no cálculo da PTF é dada por $K=\frac{\mathrm{K}}{p}$. Caso o preço relativo do capital seja estável no tempo, ou seja $p$ e $p_{K}$ cresçam à mesma taxa, a taxa de crescimento de $K$ será igual a de $K^{*}$, e não haverá nenhum problema com a medição da PTF. ${ }^{7}$ Entretanto, isto não é o que acontece no caso do Brasil.

Para avaliar o efeito do crescimento do preço relativo do capital na mediação da PTF suponha uma função de produção do tipo Cobb-Douglas, que costuma ser utilizada na maioria das estimativas de produtividade. Denotando por $A$ a PTF calculada a partir de $K$ e $A^{*}$ a PTF calculada com $K^{*}$, é possível escrever:

$$
\frac{A}{A^{*}}=\frac{Y}{K^{\theta} L^{1-\theta}} \frac{K^{* \theta} L^{1-\theta}}{Y}=\left(\frac{K^{*}}{K}\right)^{\theta}
$$

Lembrando que a diferença entre $K$ e $K^{*}$ decorre de diferenças nos preços, a equação (6) implica que a diferença entre a taxa de crescimento de $A$ e $A^{*}$ será dada por:

$$
\frac{\dot{A}}{A}-\frac{\dot{A}^{*}}{A^{*}}=\theta\left(\frac{\dot{p}}{p}-\frac{\dot{p}_{K}}{p_{K}}\right)
$$

A equação (7) explica por que o aumento do preço relativo do capital faz com que a PTF verdadeira, medida com a correção dos preços relativos, seja maior do que a PTF medida sem esta correção. Quando o preço do capital sobe mais do que o nível geral de preços, a taxa de crescimento de $A$ é menor do que a taxa de crescimento de $A^{*}$. É exatamente este o fenômeno ilustrado na Figura 8.

\section{PROGRESSO TÉCNICO ESPECÍFICO AO INVESTIMENTO}

As medidas da produtividade apresentadas acima tratam todas as gerações de capital como iguais. Como Grenwood e Jovanovic (2001) resumiram, avanços na tecnologia tendem a ser incorporados nas novas geraçôes, ou safras, de capital. Isto também

7 De fato, seria preciso que o preço relativo de cada componente do estoque de capital fosse estável. Ver Jorgenson e Griliches (1967). 
significa que pode não haver progresso técnico sem investimento. Esta abordagem é conhecida na literatura como tecnologia incorporada ao capital. Um dos primeiros modelos desenvolvidos nesta área é devido a Solow (1960), e este é próximo à versão apresentada aqui.

Novamente, suponha que a tecnologia seja caracterizada pela seguinte função de produção:

$$
Y=F(K, L)
$$

O produto pode ser utilizado para consumo $(C)$, investimento em máquinas e equipamentos $\left(X_{e}\right)$ e investimento em construçóes $\left(X_{s}\right)$. Portanto, a restrição orçamentária desta economia será dada por $C+X_{e}+X_{s}=Y=F(K, L)$. O estoque de capital será a soma do estoque de construções $\left(K_{s}\right)$ e do estoque de máquinas e equipamentos $\left(K_{e}\right)$, ou seja, $K=K_{s}+K_{e}$.

O único bem produzido pode ser usado como bem de consumo, máquina ou como estrutura. Dito de outra forma, as estruturas podem ser trocadas de um para um pelo bem de consumo ou pelas máquinas. O estoque de estruturas movimenta-se ao longo do tempo segundo a equação (2), mas com uma taxa de depreciação específica dada por $\delta_{s}$ :

$$
K_{s, t+1}=\left(1-\delta_{s}\right) K_{s, t}+X_{s, t}
$$

A acumulação de máquinas e equipamentos será descrita por uma versão modificada da regra de acumulação acima:

$$
K_{e, t+1}=\left(1-\delta_{e}\right) K_{e, t}+q X_{e, t}
$$

onde $\delta_{e}$ representa a depreciação das máquinas e dos equipamentos. Aqui o ponto mais importante é o fator $q$, que representa o estado corrente da tecnologia para produzir novos equipamentos. Este fator determina o montante de equipamento que pode ser comprado por uma unidade de produto. Quando $q$ aumenta, uma quantidade maior de bem de capital pode ser produzida com uma unidade de produto ou consumo. Mudanças em $q$ são consideradas como o progresso técnico específico ao investimento. 
No equilíbrio competitivo, o preço relativo de novos bens de equipamentos, $p$, deve ser dado por $p=1 / q$ porque isto mostra quanto de produto ou consumo de bens deve ser dado para comprar uma nova unidade de equipamento. Para identificar a mudança na tecnologia específica ao investimento, $q$, é comum utilizar-se os preços dos novos bens de capital (máquinas e equipamentos), ou seja, usando a simples relação $q=1 / p$. Um problema associado à mensuração da tecnologia incorporada no Brasil é o fato de que não possuímos uma série de preços de equipamento ajustada para a qualidade dos novos produtos na forma da série produzida por Gordon (1990) para a economia americana. Isto significa que nos períodos normais nossas séries possuem um viés negativo em relação à qualidade dos novos produtos.

A Figura 9 mostra a série de preços para os novos equipamentos e a série da tecnologia incorporada. Nos anos 70 , a taxa de crescimento de $q$ foi de $0,85 \%$, na década seguinte foi de $-0,56 \%$ e, finalmente nos anos 90 , a taxa cresceu para $5,99 \%$ ao ano.

\section{FIGURA 9 - PREÇO RELATIVO DE MÁQUINAS E EQUIPAMENTOS, $p$, e PROGRESSO TÉCNICO ESPECIFICO AO INVESTIMENTO, $q$}

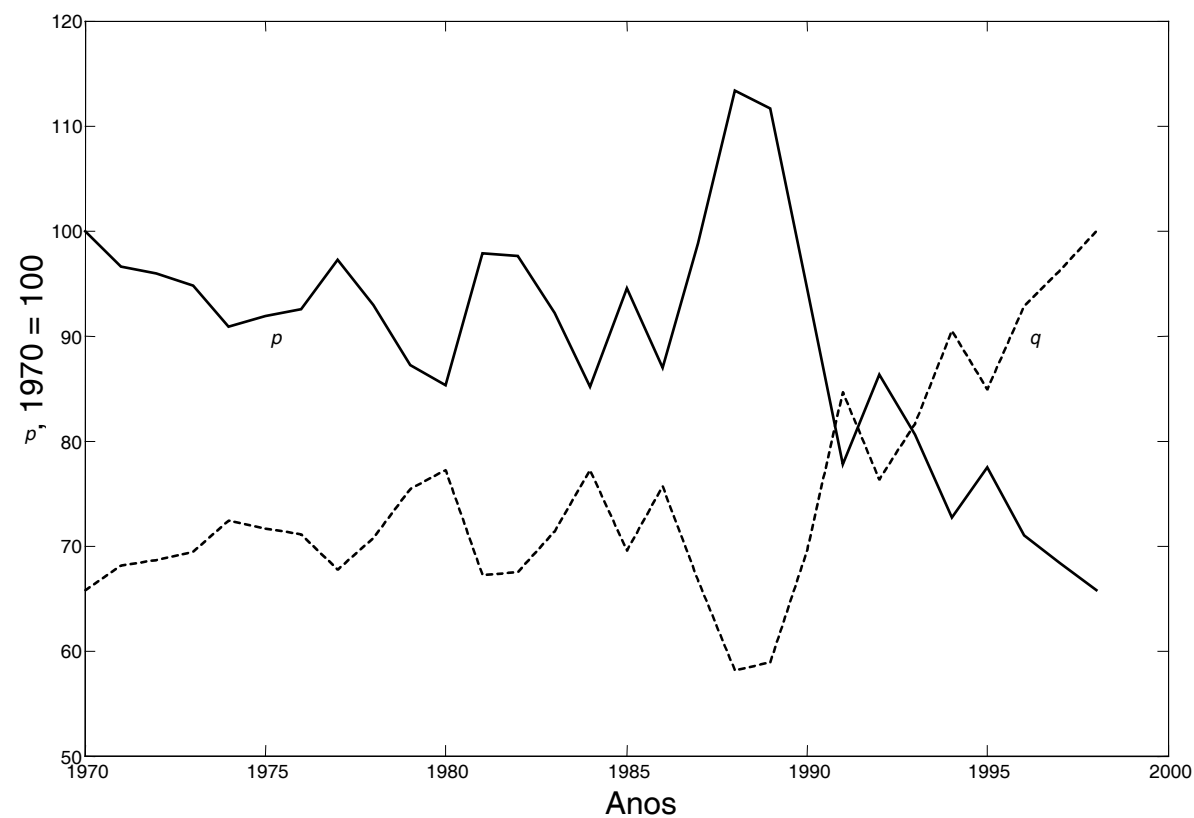

Agora torna-se possível calcular a PTF para o caso de tecnologia incorporada às máquinas e aos equipamentos. Nesta abordagem a função de produção será dada por:

$$
Y_{t}=A_{t}^{1-\theta_{e}-\theta_{s}} K_{e, t}^{\theta_{e}} K_{s, t}^{\theta_{s}} L_{t}^{1-\theta_{e}-\theta_{s}}
$$


onde $\theta_{e}$ e $\theta_{s}$ representam as participações no capital de máquinas e equipamentos e estruturas, respectivamente. Para computar a PTF devemos calibrar os parâmetros de participação do capital. O valor de $\theta_{s}$ foi calculado como a participação da renda das estruturas na renda total da economia. Como medida de renda das estruturas foi utilizado o valor dos aluguéis para os anos 70 e 90 e o valor encontrado foi $\theta_{s}=0,11{ }^{8}$ Este valor implica que $\theta_{e}=\theta-\theta_{s}=0,29$.

A Figura 10 mostra a série calculada com tecnologia incorporada e compara o resultado com o caso base. Apesar da PTF com tecnologia específica estar quase sempre acima da PTF do caso base, ambas as séries apresentam comportamento bastante semelhante. A recuperação durante os anos 90, entretanto, é bem mais acentuada.

\section{FIGURA 10 - PTF SEM TENDÊNCIA, CASO E BASE E COM TECNOLOGIA INCORPORADA}

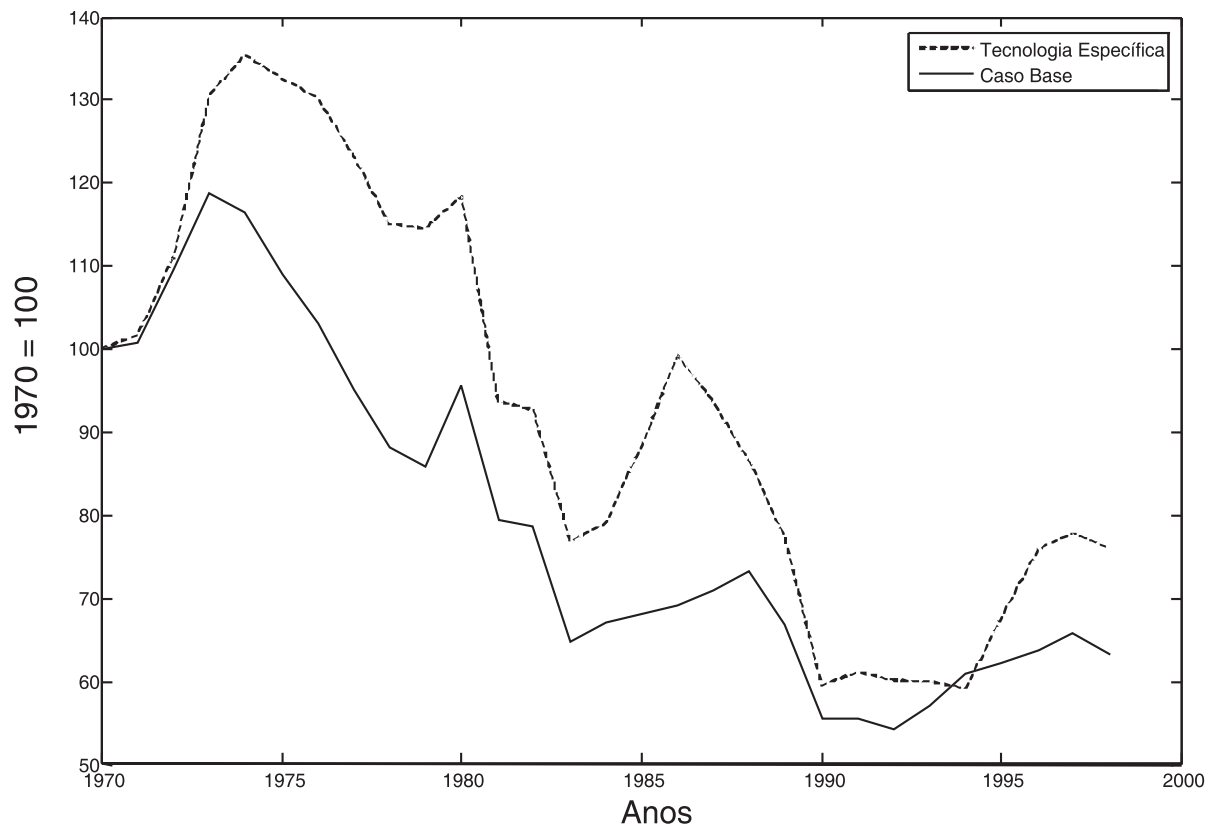

O problema que surge da utilização deste modelo de safra de capital é o mesmo destacado na literatura sobre a queda da produtividade nos Estados Unidos. ${ }^{9}$ Neste sentido, quando o progresso técnico investimento-específico aumenta, a medida da produtividade cai, e cai tanto quanto o medido pelo resíduo de Solow. Portanto, para o caso

8 Excluímos os anos 80 porque a hiperinflação nesse período reduziu muito o valor dos aluguéis em relação a seus valores históricos.

9 Para uma resenha, ver Greenwood e Jovanovic (2001). 
brasileiro, quando a tecnologia investimento-específico aumenta muito nos anos 90 , isto implica que a PTF cai proporcionalmente como no nosso caso base, justamente quando a economia está experimentando uma grande entrada de tecnologia.

\section{CONCLUSÕES}

O principal resultado encontrado é a robustez do acentuado declínio da produtividade total dos fatores no Brasil de 1970 a 2000 diante da utilização de diversas formas alternativas de mensuração da produtividade. Algumas modificações no modelo básico, como semana de trabalho do capital e do trabalho, adição de capital humano, o uso da energia como proxy para o estoque de capital e a hipótese de tecnologia específica não foram suficientes para mudar este fato empírico.

A única estimação diferente é quando a PTF é corrigida por movimentos nos preços relativos. Entretanto, mesmo neste caso, o declínio da produtividade nos anos 80 permanece. A mudança no comportamento da PTF decorrente do ajuste no preço relativo do capital pode ser interpretada como um indício de que a produtividade da economia brasileira pode ter tido uma recuperação nos anos 90 mais intensa do que o estipulado por outras medidas, em particular durante a década de 90 a PTF teria retornado à sua tendência.

Se este problema pode comprometer os exercícios de contabilidade do crescimento realizados sem a correção por preços relativos, é uma questão para futuras pesquisas. De todo modo, vale ressaltar que a queda da PTF nos anos 70 e 80 é robusta a esta especificação.

A robustez no comportamento da PTF sugere uma linha de pesquisa pouco desenvolvida no Brasil, que buscaria estudar as causas últimas da queda da produtividade a partir dos anos 70. A princípio, pensamos em três explicações para a queda da produtividade nos anos 70 e 80: (i) a intensificação de políticas protecionistas após os choques do petróleo e os problemas com balanço de pagamento no período; (ii) a crescente intervenção do Estado em setores produtivos, especialmente com o Segundo PND; (iii) e a intervenção do governo no sistema de preços relativos da economia. Pesquisas futuras devem se concentrar não só nas causas da queda da produtividade a partir de 1973, mas também porque ela não se recuperou o suficiente nos anos 90. 
APÊNDICE A. SÉRIES

\section{A.l Produto, Trabalho e Capital}

Produto - a série de PNB foi extraída do Sistema de Contas Nacionais, do Instituto Brasileiro de Geografia e Estatística (IBGE). A série foi deflacionada com o deflator implícito do produto.

Horas, emprego e semana de trabalho - séries de Bugarin et alli (2002), baseadas nos dados da Pesquisa Nacional por Amostragem Domiciliar (PNAD), IBGE. A semana de trabalho foi obtida na PNAD para o período entre 1970 e 1998. Trata-se de um valor agregado para horas médias trabalhadas por semana em atividades de mercado. A série de emprego é do Sistema de Contas Nacionais para os anos de 1991 a 1998. Para o período de 1970 a 1990 foi calculada a razão entre população empregada e população economicamente ativa (PEA) nos sensos de 1970, 1980 e 1990; depois de calculada, esta razão foi multiplicada pela PEA da PNAD.

Investimento - como série de investimento utilizamos a Formação Bruta de Capital Fixo, do Sistema de Contas Nacionais, IBGE. Esta série não inclui o consumo de bens duráveis. A partir da série agregada podemos separar o investimento em máquinas e equipamentos, estruturas (residencial e não-residencial), estoques e outros.

Estoque de capital inicial para a série de investimento-específico - o ponto inicial para a série de equipamentos foi tomado como o valor de $K_{e}$ a partir da trajetória de crescimento equilibrado do modelo para o ano de 1970. A equação que descreve esta relação é a seguinte:

$$
K_{e}=\frac{q i_{e}}{\left(g_{y}+1\right)\left(g_{q}+1\right)-\left(1-\delta_{e}\right)}
$$

onde $g_{y}$ representa a taxa de crescimento do produto real, $g_{q}$ a taxa de crescimento da tecnologia embutida e $i_{e}$ o investimento fixo privado nominal em máquinas e equipamentos deflacionado pelo índice de preços do consumo pessoal de não-duráveis e serviços não-domésticos (a respeito do uso deste índice, ver Greenwood e Jovanovic, 1991, p. 218). O valor utilizado para $g_{q}$ foi de $4 \%$, igual ao observado nos Estados Unidos desde 1974.

Utilização do capital - taxa anual média de utilização do capital, da Fundação Getúlio Vargas, 1970-1998. 
Consumo de Energia - a série utilizada é o consumo de energia elétrica industrial, do Ministério das Minas e Energia, 1970-1998.

Capital humano - utilizamos os dados fornecidos por Barro e Lee (2000). A série original é qüinqüenal; para criar uma série de tempo para todo o período realizamos interpolação linear para os anos intermediários.

\section{A.2 Preços}

Como relatado em Greenwood, Hercowitz e Krussel (1997, p. 360), devemos evitar problemas com números índices para contabilizar o progresso tecnológico no setor produtor de equipamentos. Portanto, não podemos usar as medidas-padrão de produto e equipamentos para medir estas séries no caso da tecnologia específica ao investimento. O modelo de tecnologia específica ao investimento deve ter suas quantidades expressas em termos do seu custo em unidades de consumo.

Assim, produto e investimento em máquinas e equipamentos, e também o investimento em estruturas, foram deflacionados usando uma série de preços que representasse melhor os preços dos bens de consumo. Infelizmente, o Brasil não possui um índice de preços ao consumidor longo o suficiente para o nosso trabalho. Portanto, na prática usamos o IGP-DI que possui 30\% da composição como índice de preços ao consumidor.

IGP-DI - Índice Geral de Preços - índice de preços da economia para as mercadorias e serviços produzidos domesticamente, elaborado pela Fundação Getúlio Vargas (FGV) desde 1944. O índice é uma média ponderada do IPA-DI, preço de venda ao atacado para os produtos e serviços domésticos (peso de 60\%), índice de preços ao consumidor (30\%) e índice de preços da construção civil, INCC (10\%).

IPA-OG para Máquinas e Equipamentos - índice de preços ao atacado cobrindo a produção doméstica e os bens importados. Elaborado pela FGV.

IPA-DI para Materiais de Construção - índice de preços ao atacado cobrindo a produção doméstica. Elaborado pela FGV.

\section{APÊNDICE B. PRODUTIVIDADE E TENDÊNCIAS}

Seguindo Prescott (2002) e a literatura relacionada ao estudo de depressões, retiramos a tendência de crescimento da fronteira tecnológica dada pela economia líder. Como 
economia líder usamos os EUA, pois é o país líder de crescimento no século 20. Para descontar a performance da economia líder, assumimos que ela está na trajetória de crescimento equilibrado e, portanto, crescendo a uma taxa constante. A taxa de crescimento média do século 20 do produto por pessoas em idade de trabalho foi de $2 \%$ ao ano. Assumindo que o crescimento dos EUA é dado por tecnologia trabalho-aumentativa, isto implica que $g_{y}=g_{a}^{1-\theta}$, portanto a taxa de crescimento média da produtividade, $g_{a}$, é de $1: 44 \%$. Assim, retiramos a tendência das séries de PTF da seguinte forma:

$$
B_{t}^{d}=\frac{B_{t}}{B_{1970}\left(1+g_{a}\right)^{t-1969}}
$$

onde $B_{t}^{d}$ é a série sem tendência, $B_{t}$ é a série com tendência e $t=\{1970,1971, \ldots, 1998\}$ é o tempo.

\section{REFERÊNCIAS BIBLIOGRÁFICAS}

Abreu, Marcelo de Paiva (ed.). A ordem do progresso: cem anos de politica econômica. Rio de Janeiro: Campus, 1990.

Barro, Robert J.; Lee, Jong-Wha. International data on educational attainement: updates and implications. NBER Working Paper No. 7911, 2000.

Bils, Mark J.; Klenow, Peter J. Does schooling cause growth? American Economic Review, v. 90, n. 5, p. 1160-1183, 2000.

Bonelli, Regis; Fonseca, Renato. Ganhos de produtividade e eficiência: novos resultados para a economia brasileira. Pesquisa e Planejamento Econômico, v. 28, n.2, 1998.

Bugarin, Mirta; Ellery Jr, Roberto; Gomes, Victor; Teixeira, Arilton. The Brazilian depression in the 80s and 90s. In: Kehoe, Timothy J.; Prescott, Edward C. (eds.). Great depressions of the twentieth century. Minneapolis: Federal Reserve Bank of Minneapolis, 2007.

Ferreira, Pedro C.; Issler, João Victor; Pessôa, Samuel A. Testing production functions used in empirical growth studies. Economic Letters, v. 83, n. 1, p. 29-35, April 2004.

Ferreira, Pedro C.; Malliagros, Thomas G. Investimentos, fontes de financiamento e evolução do setor de infra-estrutura no Brasil. Ensaios Econômicos da EPGE, 346. Rio de Janeiro: Fundação Getúlio Vargas, 1999.

Gollin, Douglas. Getting income shares right. Journal of Political Economy, v. 110, n. 2, p. 458-74, 2002. 
Gomes, Victor; Bugarin, Mirta; Ellery Jr, Roberto. Long-run implications of the Brazilian capital stock and income estimates. Brazilian Review of Econometrics, v. 25, n. 1, 2005.

Gomes, Victor; Pessôa, Samuel; Veloso, Fernando. Evolução da produtividade total dos fatores na economia brasileira: uma análise comparativa. Pesquisa e Planejamento Econômico, v. 33, n. 3, p. 389-434, 2003.

Gomes, Victor; Lisboa, Marcos B.; Pessôa, Samuel A. Estudo da evolução da produtividade total dos fatores na economia brasileira: 1950-2000. 2002. (Manuscrito).

Gordon, Robert J. The measurement of durables goods prices. Chicago: Chicago University Press, 1990.

Greenwood, Jeremy; Hercowitz, Zvi; Krussell, Per. Long-Run implications of investment-specific technological change. American Economic Review, v. 87, n. 3, p. 342-362, 1997.

Greenwood, Jeremy; Jovanovic, Boyan. Accounting for growth. In: Dean, Edwin; Harper, Michael; Hulten, Charles (eds.). New directions in productivity analysis. Chicago: Chicago University Press (for NBER), 2001.

Jorgenson, Dale W.; Griliches, Zvi. The explanation of productivity change. Review of Economic Studies, v. 34, n. 3, p. 249-283, 1967

Kydland, Finn; Prescott, Edward. Time to build and aggregate fluctuations. Econometrica, v. 50, n.6, p. 1345-1370, 1982.

Kydland, Finn; Zarazaga, Carlos. Argentina's lost decade and subsequent recovery puzzle. In: Kehoe, Timothy J.; Prescott, Edward C. (eds.). Great depressions of the twentieth century. Minneapolis: Federal Reserve Bank of Minneapolis, 2007.

Ohanian, Lee E. Why did productivity fall so much during the Great Depression? American Economic Review, v. 91, n. 2, p. 34-38, 2001.

Pinheiro, Armando C.; Gill, Indermit; Serven, Luis; Thomas, Mark R. Brazilian economic growth, 1900-2000: lessons and policy implications. Unpublished manuscript, 2001.

Prescott, Edward C. Business cycle research: methods and problems. Federal Reserve Bank of Minneapolis, 1998. (Working Paper Number 590). 2, p. 1-15, 2002.

Prosperity and depression. American Economic Review, v. 92, n.

Pritchett, Lant. The tyranny of concepts: CUDIE (cumulated, depreciated, investment effort) is not capital. Journal of Economic Growth, v. 5, p. 361-384, 2000.

Solow, Robert M. Technical change and the aggregate production function. The Review of Economics and Statistics, v. 39, n. 3, p. 312-320, 1957.

. Investment and technological progress. In: Arrow, Kenneth; Karlin, Samuel; Suppes, Patrick (eds.) Mathematical methods in the social sciences 1959. Stanford: Stanford University Press, 1960. 